\title{
Comparative Studies on Lipid Analysis and Ultrastructure in Porcine and Southern Minke Whale (Balaenoptera Bonaerensis) Oocytes
}

\author{
Takuma FUJIHIRA ${ }^{1)}$, Mikio KINOSHITA ${ }^{2)}$, Motoki SASAKI ${ }^{3)}$, \\ Masao OHNISHI ${ }^{2}$, Hajime ISHIKAWA ${ }^{4)}$, Seiji OHSUMI ${ }^{4)}$ and Yutaka FUKUI ${ }^{1)}$ \\ ${ }^{1)}$ Laboratory of Animal Reproduction, ${ }^{2)}$ Laboratory of Applied Bioscience, ${ }^{3)}$ Laboratory of \\ Veterinary Anatomy, Obihiro University of Agriculture and Veterinary Medicine, Obihiro 080- \\ 8555, ${ }^{4)}$ The Institute of Cetacean Research, Tokyo 104-0055, Japan
}

\begin{abstract}
The present study was conducted to clarify the difference in the color of the cytoplasm in immature follicular oocytes from prepubertal and adult minke whales. The four lipid contents (triglyceride, total cholesterol, phospholipids and non-esterified fatty acids) in vitrified immature oocytes from prepubertal and adult minke whales, and also in fresh and vitrified immature porcine oocytes, were measured. The lipid contents in vitrified-warmed minke whale oocytes were similarly high compared with those in vitrified-warmed porcine oocytes. In particular, the total cholesterol and phospholipid contents in the vitrified immature oocytes from prepubertal and adult minke whales were significantly $(\mathrm{P}<0.05)$ higher than those from prepubertal pigs. Furthermore, the distribution of lipid droplets in fresh and vitrified immature oocytes was observed in transmission electron microscopy. Lipid droplets in the prepubertal minke whale oocytes were distributed throughout the cytoplasm. In contrast, adult minke whales had larger lipid droplets which were distributed mainly in the central portion of the cytoplasm. The lipid droplets of immature oocytes from prepubertal pigs were larger than those in minke whale oocytes. These results indicated that the difference in the distribution of the cytoplasmic lipid droplets may result in the difference in the color tone of both prepubertal and adult whale oocyte cytoplasm.
\end{abstract}

Key words: Minke whale, Porcine, Oocytes, Lipid, Sexual maturity

(J. Reprod. Dev. 50: 525-532, 2004)

Ш have been studying in vitro maturation (IVM), fertilization (IVF) and culture (IVC) in the artificial production of minke whale embryos [1-4]. During the studies, we observed a difference in the color of the cytoplasm in immature follicular oocytes from prepubertal and adult minke whales. The cytoplasm of the oocytes collected from prepubertal whales was opaque and dark, whereas the oocytes from adult whales had bright and transparent cytoplasm (Fig. 1). It was considered

Accepted for publication: May 17, 2004

Correspondence: Y. Fukui (e-mail: fukui@obihiro.ac.jp) that the difference in the color of the two oocyte types was related to the lipid content in the cytoplasm. Lipids in the oocytes are mainly involved in energy storage, constitute the cytoplasmic membrane and cytoplasmic organelles, and adjust the physiological function. Lipid analyses in oocytes or embryos have been conducted in domestic animals [5-8], and it was reported that porcine oocytes had a dark color tone of the cytoplasm as well as minke whale oocytes, and porcine oocytes had higher lipid content than the oocytes of other species [7].

Follicular oocytes of many species are very 


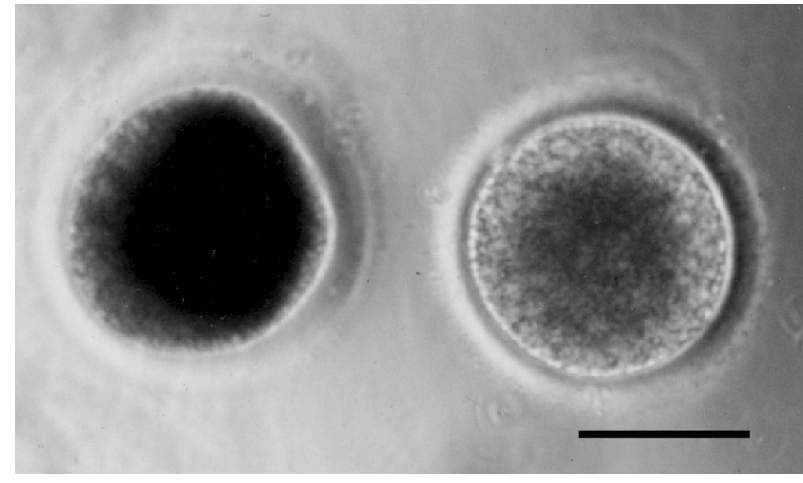

Fig. 1. Immature oocytes from prepubertal (left) and adult (right) minke whales. The former cytoplasm is dark and opaque, and the latter cytoplasm is bright and transparent. Bar: $100 \mu \mathrm{m}$.

sensitive to low temperature, and their survivability after chilling or freezing is low. Didion et al. [9] reported that porcine oocytes did not survive after cooling to lower than 15 degrees, and the in vitro maturation rates of frozen-thawed porcine and minke whale oocytes were significantly lower than those of other species. It seems that one of the reasons why porcine and minke whale oocytes have low cryotolerance is likely due to the high intracellular lipids. Libermann et al. [10] reported that intracellular lipids caused deformation and disruption of the cytoskeleton during cooling and vitrification.

$\mathrm{O}^{\prime}$ Brien et al. [11] reported that the size of mitochondria and cortical granules in oocytes from adult sheep was larger than that in oocytes from prepubertal sheep. Significant differences in nuclear maturation and developmental competence were found between the oocytes from prepubertal and adult animals in several species including pigs [12] and cattle [13, 14]. Nevertheless, there seem to have been no reports on comparative studies on lipid content and distribution of lipid droplets in follicular oocytes from adult and prepubertal animals. If the oocytes from prepubertal animals have a higher content of lipids than oocytes from adult animals, the oocytes from prepubertal animals may have less cryopreservation ability than those from adult animals.

We hypothesized in the present study that the characteristics (dark color and opaqueness) of the cytoplasm of the prepubertal minke whale were related to the higher lipid content and lipid distribution than that of the adult minke whale. In order to clarify the hypothesis, we examined the lipid content in vitrified immature oocytes from prepubertal and adult minke whales, and in fresh and vitrified immature porcine oocytes. In addition, the distribution of lipid droplets in fresh and vitrified immature oocytes was also observed by transmission electron microscopy (TEM).

\section{Material and Methods}

The present study was approved by the Animal Experimental Committee of Obihiro University of Agriculture and Veterinary Medicine, in accordance with the Guiding Principles for the Care and Use of Research Animals.

\section{Animals and oocyte collection}

The southern minke whales used in this study were captured during the period from November 2001 until March 2002 in the Japanese Whale Research Programme with a Special Permit for the Antarctic (JARPA) and was organized by the Institute of Cetacean Research in Tokyo, Japan. A total of 207 female southern minke whales (prepubertal: $\mathrm{n}=76,6.72 \pm 0.97$ meters, $3.99 \pm 1.51$ tons; adult: $\mathrm{n}=131,8.97 \pm 0.39$ meters, $8.26 \pm 1.19$ tons) were captured in the area $60-68^{\circ} \mathrm{S}$ and 35 $130^{\circ} \mathrm{E}$. All the whales used in the present study were killed by explosive harpoons which have been recognized as the most humane killing method for whales by the International Whaling Commission and provided for by Schedule III (Capture) in the International Convention for the Regulation of Whaling. Special attention to reduce the time to death was given to all sampled whales. Explosive harpoons were used for all whales as the primary killing method. A large-caliber rifle was used as the secondary killing method when required. Ovaries were collected within $3 \mathrm{~h}$ after death and were kept warm at $20 \mathrm{C}$ until oocyte collection (within $10 \mathrm{~h}$ ).

Sexual maturity of the minke whale was determined by the presence or absence of a corpus luteum and/or corpora albicans on either ovary. Whales with neither corpus luteum nor corpus albicans were considered prepuberal.

Minke whale oocytes were aspirated from follicles ( 2 to $15 \mathrm{~mm}$ in diameter) by using a 10-ml syringe fitted with an 18-gauge needle. The syringe contained modified PBS (m-PBS; Embryo Tech; 
Nihon-Zenyaku Industrial Co., Kohriyama, Japan) supplemented with $0.9 \%(\mathrm{v} / \mathrm{v})$ sodium citrate solution (Citramin; Fusou Pharmaceutical Co., Osaka, Japan). Oocytes surrounded by more than one layer of cumulus cells and with homogeneous cytoplasm were selected and washed three times with m-PBS. Until vitrification (within $1 \mathrm{~h}$ ), the oocytes were kept on m-PBS at $39 \mathrm{C}$. One thousand two hundred each of prepubertal and adult minke whale oocytes were used in this study.

Porcine follicular oocytes were collected from prepubertal (6 months old) gilts at a local abattoir and transported within $3 \mathrm{~h}$ to the laboratory in $0.9 \%$ $(\mathrm{w} / \mathrm{v}) \mathrm{NaCl}$ at 30-35 C. Oocytes were aspirated from follicles ( 3 to $6 \mathrm{~mm}$ in diameter), and were selected and subjected to three washes in Hepes buffered Tyrode's medium containing $0.1 \%(\mathrm{w} / \mathrm{v})$ polyvinyl alcohol (PVA; Sigma Chemical Co., St. Louis, MO, USA)(TLH-PVA). Porcine oocytes were kept on TLH-PVA at $39 \mathrm{C}$ until vitrification (within $1 \mathrm{~h}$ ). A total of 2,750 porcine oocytes were used in this study.

\section{Vitrification and warming}

The basic medium used for vitrification was dulbecco's PBS (D-PBS; Nissui pharmaceutical Co., Tokyo, Japan) supplemented with $20 \%(\mathrm{v} / \mathrm{v})$ newborn calf serum (NBCS). After three washes in the basic medium, the oocytes were equilibrated to $15 \%(\mathrm{v} / \mathrm{v})$ ethylene glycol (EG) and $0.25 \mathrm{M}$ sucrose for $4 \mathrm{~min}$ at room temperature (20-25 C), and then transferred to $40 \%$ EG containing $0.5 \mathrm{M}$ sucrose and were equilibrated for $90 \mathrm{sec}$. The oocytes were immediately loaded into an open pulled straw (OPS) and vitrified in liquid nitrogen. For warming, the OPS was exposed to air at room temperature $(20-25 \mathrm{C})$ for $5 \mathrm{sec}$ before being immersed in $30 \mathrm{C}$ water for $10 \mathrm{sec}$. The oocytes were exposed to $0.5 \mathrm{M}, 0.25 \mathrm{M}$, and $0.125 \mathrm{M}$ sucrose solutions in mPBS for $0.5,5$, and $5 \mathrm{~min}$, respectively. They were then transferred to the basic medium and morphologically intact oocytes were selected.

\section{Total lipid extraction}

Total lipids of the vitrified-warmed immature oocytes from the prepubertal and adult minke whales and of the fresh and vitrified-warmed immature oocytes from prepubertal pigs were extracted by the method of Bligh and Dyer [15]. Cumulus cells surrounding oocytes were denuded by vortexing for $5 \mathrm{~min}$ in $1 \%$ hyaluronidase and by vigorous pipetting. Denuded oocytes were transferred to $1.0 \mathrm{ml}$ PBS (200 to 1000 oocytes $/ \mathrm{ml}$ ). They were then added immediately to $2 \mathrm{ml}$ methanol and $1 \mathrm{ml}$ chloroform and sonicated for 10 min. After sonication, $1 \mathrm{ml}$ chloroform and $0.8 \mathrm{ml}$ water were added to the adulterant followed by centrifugation at $1500 \mathrm{~g}$ for $10 \mathrm{~min}$. The lower chloroform layer was taken out and evaporated to dryness under nitrogen. The residuals containing total lipids were added to $100 \mu$ 2-propanol.

\section{Lipid assay}

The four lipids were measured as follows: for assay of triglyceride, total cholesterol, phospholipids and non-esterified fatty acids, the triglyceride E test, total cholesterol E test, phospholipids $\mathrm{C}$ test and non-esterified fatty acids $\mathrm{C}$ test were used, respectively. For each assay, $5 \mu \mathrm{l}$ out of $100 \mu \mathrm{l} 2$-propanol containing the total lipids were examined. Colorimetric assays were conducted on microplates and read at the appropriate wavelengths $(600 \mathrm{~nm}$ for triglyceride, total cholesterol and phospholipids and $550 \mathrm{~nm}$ for non-esterified fatty acids) on a microtitre plate spectrophotometer.

The results of lipid analysis were expressed by two kinds of units; pmol per oocyte and $10^{-5} \mathrm{pmol}$ per $\mu \mathrm{m}^{3}$. The mean oocyte diameters $(\mathrm{n}=10)$ were $164 \mu \mathrm{m}, 198 \mu \mathrm{m}$ and $180 \mu \mathrm{m}$ for porcine, prepubertal whale and adult whale, respectively.

\section{TEM preparation}

The porcine and minke whale oocytes were fixed in $2.5 \%$ glutaraldehyde for $2 \mathrm{~h}$ and in $1 \%$ osmium tetraoxide for $1 \mathrm{~h}$. After dehydration with ethanol, the oocytes were embedded in Spurr's epoxy resin. Ultrathin sections $(75 \mathrm{~nm})$ were cut out with an ultramicrotome and double-stained with $4 \%$ uranyl acetate and $0.5 \%$ lead citrate. The characteristics of the lipid distribution in the minke whale and porcine oocyte cytoplasm were examined with an H-7500 transmission electron microscope (Hitachi, Tokyo, Japan).

\section{Statistical analysis}

One sample (200 to 1,000 oocytes) was used to analyze the four kinds of lipid contents in three replicates. The lipid contents of the vitrifiedwarmed immature oocytes from prepubertal and adult minke whales and of the fresh and vitrified- 
Table 1. Lipid contents (upper; pmol per oocyte, lower; $10^{-5}$ pmol per $\mu \mathrm{m}^{3}$ ) of vitrified-warmed immature oocytes from prepubertal and adult minke whales and of fresh, and vitrified-warmed immature oocytes from prepubertal pigs

\begin{tabular}{ccccccc}
\hline \multirow{2}{*}{ Animal } & Sexual maturity & Fresh/vitrified & Triglyceride & Total cholesterol & Phospholipid & $\begin{array}{c}\text { Non-esterified } \\
\text { fatty acid }\end{array}$ \\
\hline \multirow{3}{*}{ Whale } & \multirow{2}{*}{ Prepubertal } & \multirow{2}{*}{ Vitrified } & $71.2 \pm 1.6^{\mathrm{a}}$ & $61.1 \pm 3.9^{\mathrm{a}}$ & $65.7 \pm 1.4^{\mathrm{a}}$ & $103.0 \pm 3.6^{\mathrm{a}}$ \\
\cline { 2 - 6 } & \multirow{2}{*}{ Adult } & \multirow{2}{*}{ Vitrified } & $75.9 \pm 4.5^{\mathrm{a}}$ & $44.1 \pm 2.5^{\mathrm{b}}$ & $50.6 \pm 2.1^{\mathrm{b}}$ & $90.4 \pm 5.4^{\mathrm{a}}$ \\
\cline { 3 - 7 } & & $2.49 \pm 0.15^{\mathrm{y}}$ & $1.45 \pm 0.08^{\mathrm{x}}$ & $1.66 \pm 0.07^{\mathrm{x}}$ & $2.96 \pm 0.18$ \\
\hline \multirow{2}{*}{ Pig } & \multirow{2}{*}{ Prepubertal } & \multirow{2}{*}{ Vitrified } & $57.7 \pm 1.5^{\mathrm{b}}$ & $20.4 \pm 3.4^{\mathrm{c}}$ & $19.9 \pm 2.6^{\mathrm{c}}$ & $52.0 \pm 3.3^{\mathrm{b}}$ \\
\cline { 2 - 6 } & \multirow{2}{*}{ Prepubertal } & \multirow{2}{*}{ Fresh } & $2.50 \pm 0.06^{\mathrm{y}}$ & $0.88 \pm 0.15^{\mathrm{y}}$ & $0.86 \pm 0.11^{\mathrm{y}}$ & $2.25 \pm 0.14$ \\
\hline & & $67.4 \pm 1.5^{\mathrm{c}}$ & $33.5 \pm 2.0^{\mathrm{d}}$ & $16.3 \pm 1.0^{\mathrm{c}}$ & $68.2 \pm 2.9^{\mathrm{c}}$ \\
& & $2.92 \pm 0.07^{\mathrm{z}}$ & $1.45 \pm 0.09^{\mathrm{x}}$ & $0.71 \pm 0.04^{\mathrm{y}}$ & $2.96 \pm 0.12$ \\
\hline
\end{tabular}

Values are means \pm SEM of three replicates consisting of 200 to 1000 oocytes.

$a-d, x-z$ Values in the same row with different superscripts significantly differ $(P<0.05)$.

warmed immature oocytes from prepubertal pigs were compared by one-way ANOVA and Student's $t$ test.

\section{Results}

\section{Lipid contents}

Results on lipid content expressed in both units per oocyte and per volume are shown in Table 1. In units per oocyte, triglyceride contents in the vitrified immature oocytes from prepubertal and adult minke whales were significantly $(\mathrm{P}<0.05)$ higher than those from prepubertal pigs (prepubertal whales: $71.2 \pm 1.6$ pmol per oocyte, adult whales: $75.9 \pm 4.5$ pmol per oocyte, prepubertal pigs: $57.7 \pm 1.5$ pmol per oocyte). But when the lipid contents were expressed by per volume, the vitrified immature oocytes from prepubertal minke whales were significantly $(\mathrm{P}<0.05)$ lower than those from adult whales and prepubertal pigs (prepubertal whales: $1.75 \pm 0.04 \times$ $10^{-5}$ pmol per $\mu \mathrm{m}^{3}$, adult whales: $2.49 \pm 0.15 \times 10^{-5}$ pmol per $\mu \mathrm{m}^{3}$, prepubertal pigs: $2.50 \pm 0.06 \times 10^{-5}$ pmol per $\mu \mathrm{m}^{3}$ ). On the total cholesterol and phospholipid contents expressed by per oocyte and per volume, the vitrified immature oocytes from prepubertal and adult minke whales were significantly $(\mathrm{P}<0.05)$ higher than those from prepubertal pigs. For non-esterified fatty acid content, when the unit was expressed by per oocyte, the counts of vitrified immature oocytes from prepubertal and adult minke whales were significantly $(\mathrm{P}<0.05)$ higher than those from prepubertal pigs. Nevertheless, when the unit per volume was used, no significant difference was found among the vitrified immature oocytes from prepubertal and adult minke whales, and prepubertal pigs. Triglyceride and total cholesterol content in the vitrified immature porcine oocytes were significantly $(\mathrm{P}<0.05)$ lower than those in the fresh immature porcine oocytes.

\section{Ultrastructural observations}

The immature oocytes from prepubertal minke whales had a great deal of smaller lipid droplets in the cytoplasm (Fig. 2) and, these droplets were distributed throughout the cytoplasm. In contrast, the adult whale oocytes had a larger lipid droplets, which were distributed mainly in the central portion of the cytoplasm (Fig. 3). The lipid droplets of the immature oocytes from prepubertal pigs were larger than those of the minke whale oocytes, but the number of lipid droplets was less than that in the minke whale oocytes (Fig. 4). When the fresh immature and vitrified-warmed porcine oocytes were compared, the color tone of the lipid droplets was different: fresh oocytes were gray and vitrified oocytes were slightly dark (Figs. 4 and 5). Otherwise, there was no difference between the size or the distribution of lipid droplets in the fresh and vitrified-warmed porcine oocytes. 


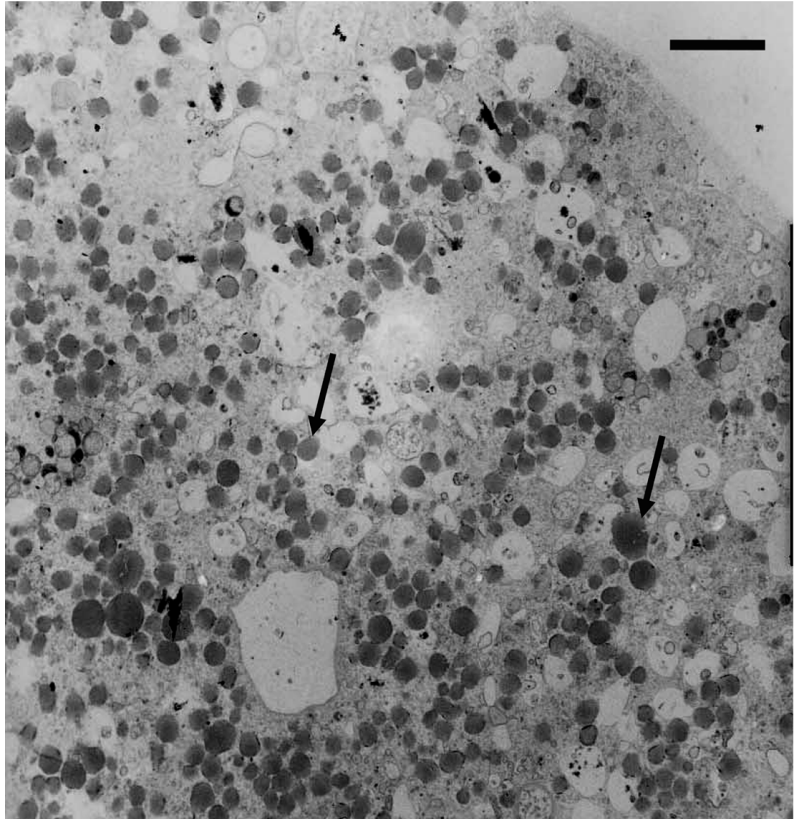

Fig. 2. A prepubertal minke whale oocyte after vitrification/ warming. Lipid droplets were distributed throughout the cytoplasm (arrows). Bar: $4.5 \mu \mathrm{m}$.

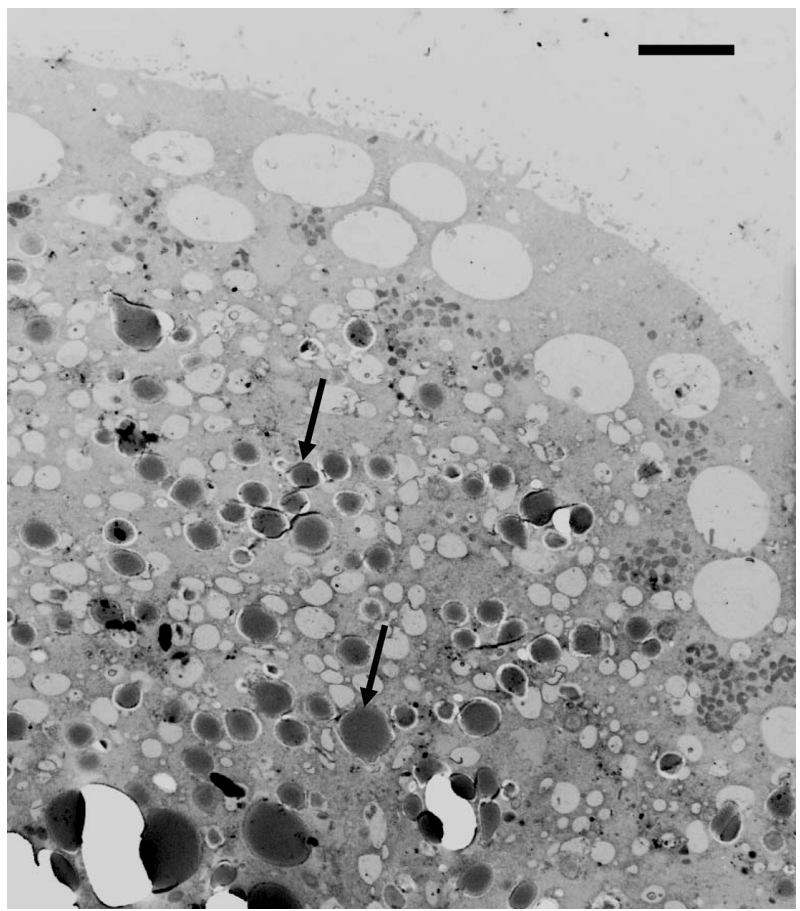

Fig. 3. An adult minke whale oocyte after vitrification/ warming. Lipid droplets were distributed mainly in the central portion of the cytoplasm (arrows). Bar: $4.5 \mu \mathrm{m}$.

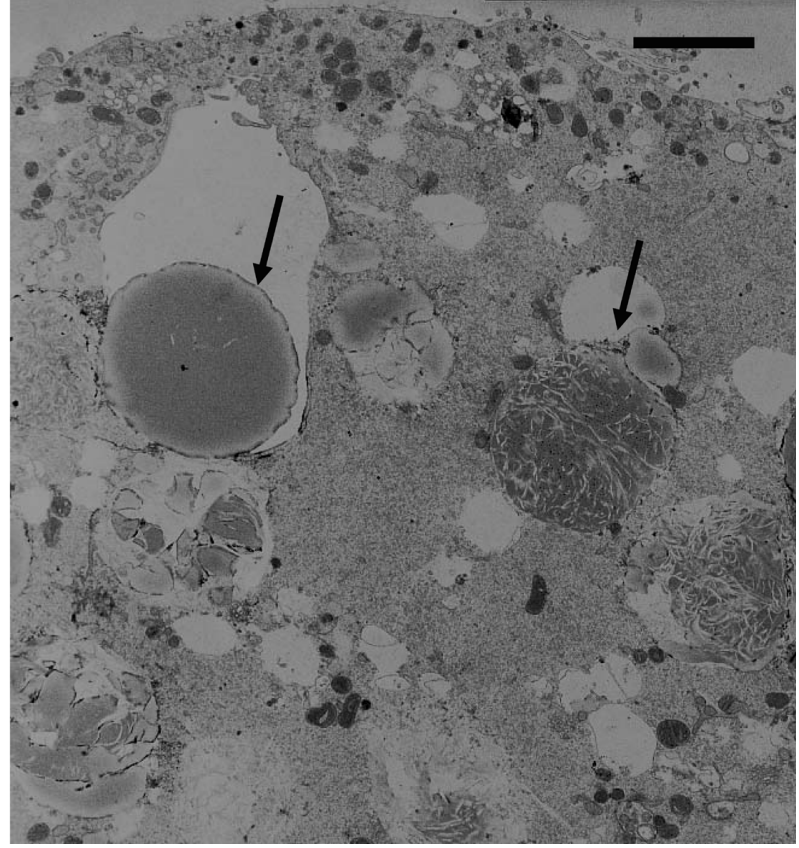

Fig. 4. Large lipid droplets were observed in a vitrified, warmed porcine oocyte. Bar: $3.0 \mu \mathrm{m}$.

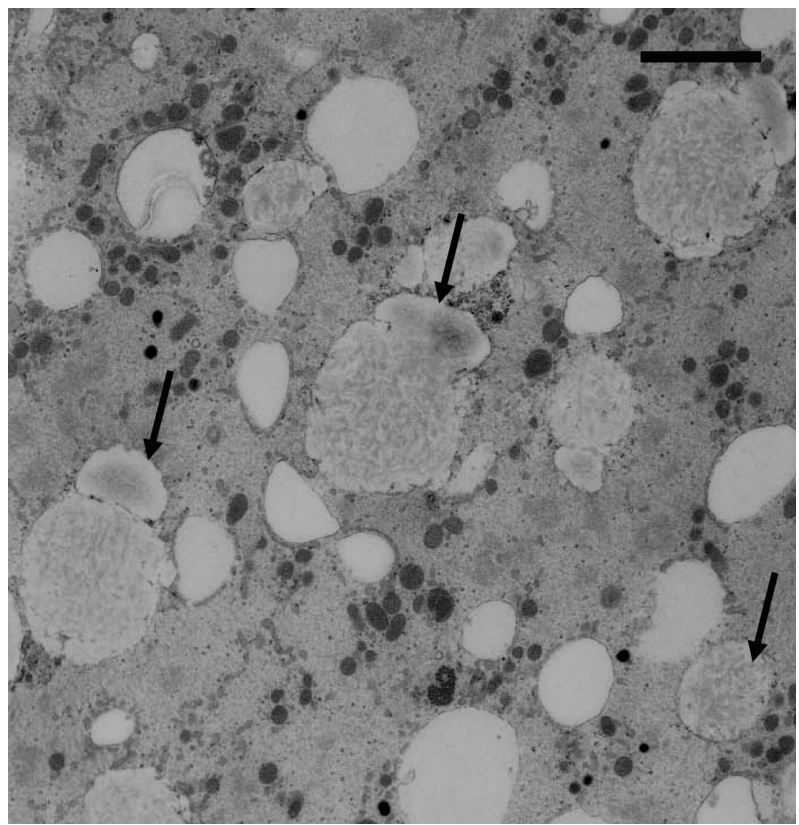

Fig. 5. Pale lipid droplets were observed in a fresh porcine oocyte. Bar: $3.0 \mu \mathrm{m}$. 


\section{Discussion}

There has been a report that porcine oocytes have higher lipid content than those of several other species [7]. Porcine oocytes were sensitive to cryopreservation, probably due to the high cytoplasmic lipids. Lipid droplets interact with the elements of the cytoskeleton in the oocytes. These interactions are destroyed during cryopreservation $[16,17]$, which causes irreversible damage to the oocyte. In the present study, the lipid contents were expressed both per oocyte and per volume as shown in Table 1. The results for triglyceride content were opposite in the vitrified whale and porcine oocytes. The significant differences in the non-esterified fatty acid expressed as units per oocyte disappeared when the units per volume was used, but total cholesterol and phospholipid contents showed similar results in both units per oocyte and per volume. These results showed that lipid content in the vitrified immature whale oocytes was as high as in vitrified immature porcine oocytes. In particular, the contents of total cholesterol and phospholipid in both prepubertal and adult minke whale oocytes were significantly $(\mathrm{P}<0.05)$ higher than those from porcine oocytes. Asada et al. [3] reported that only $3.8 \%$ of minke whale GV oocytes matured after slow freezing and thawing. Additionally, maturation rates of prepubertal and adult whale oocytes after vitrification with cryotop were $10 \%$ and $30 \%$, respectively (unpublished data). On the other hand, maturation rates of porcine oocytes after vitrification with cryotop were $45 \%$ (unpublished data), although the vitrification methods were different for whale and porcine oocytes (cryoprotectant, exposure time in equilibration solution and warming methods). Therefore, it may be speculated that minke whale oocytes are similarly sensitive to freezing or vitrification as with porcine oocytes. In order to improve cryopreservation of minke whale oocytes, further studies were required on the use of chemicals, such as cytochalasin, which have a flexible effect on cytoskeletal elements and are consequently unsusceptible to the effects of lipids [18]. Alternatively, Nagashima et al. [19, 20] obtained high survival rates of porcine embryos by polarization and removal of cytoplasmic lipids before vitrification.

It is generally recognized that the color tone of the cytoplasm looks dark, due to the many lipid droplets in it. In the present study, it was observed that lipid droplets in the prepubertal whale oocytes were distributed throughout the cytoplasm, compared with adult whale oocytes, in which they were distributed mainly in the central portion of the cytoplasm. This result may speculate that lipid droplets in the oocytes are present throughout the cytoplasm when the whale is young, and the droplets become concentrated in the central portion of the cytoplasm as the whale matures. And, the difference in the distribution of the cytoplasmic lipid droplets may result in the difference in the color tone of both prepubertal and adult whale cytoplasm. Nevertheless, the factors for the difference in the color tone of the cytoplasm could not be specified from the present study, because other materials such as pigments, besides lipids, may be related to the color tone of the cytoplasm.

$\mathrm{O}^{\prime}$ Brien et al. [11] reported that the mitochondria and cortical granules in adult sheep oocytes were larger than those in prepubertal sheep oocytes, and the rate of developmental to the blastcyst stage was lower in prepubertal sheep oocytes than in adult sheep oocytes. Their findings were likely caused by the different characteristics in the cytoplasm of prepubertal and adult sheep oocytes [23]. Nevertheless, Asada et al. [4] found no differences in the proportions of maturation, penetration and pronuclei formation between prepubertal and adult minke whale oocytes. The in vitro maturation and fertilization techniques for minke whale oocytes are still incomplete, and the maturation rate of the minke whale oocytes is lower (less than 30\%) than that of other domestic animals $[1,2,4]$.

After immature oocytes were cooled or frozen, the lipid contents within bovine oocytes decreased [8], and the ultrastructure of lipid droplets changed [24]. In the present study, triglyceride and total cholesterol contents significantly decreased when immature porcine oocytes were vitrified and warmed. It was considered from this result that the reduction in these lipids was most likely caused by cryopreservation, and these lipids might have been destroyed when the cytoplasm or cytoplasmic membrane was damaged during the vitrification procedures. Consequently, destroyed lipids have become impossible to analyze by the method used in this study. Therefore, the survivability of oocytes after freezing and thawing could be enhanced by improving the cryopreservation 
method causing a decrease in the lipid contents of oocytes. And, the color tone of the lipid droplets was different in the fresh and cryopreserved immature porcine oocytes. The difference in the color tone was most likely caused by the unsaturated fatty acid composition, although, in this study, the unsaturated fatty acid composition was not analyzed. Further studies on the fatty acid composition of prepubertal and adult oocytes are required. On the other hand, it has been reported that phase transition in the cytoplasmic lipids of immature bovine oocytes occurs between 13 and 20 degrees [25]. Holding the immature oocytes at phase transition temperature may induce more damage to the cellular membrane than exposure to lower temperatures. Likewise, Isachenko et al. [24] showed that the ultrastructure of cytoplasmic lipid droplets in immature porcine oocytes was changed by cooling the oocytes for $20 \mathrm{~min}$ at 10 degrees, but the present study has not agreed with their reports. From our results, it appears that the ultrastructural change in the lipid droplets could be caused by holding at the lipid phase transition temperature; when oocytes are frozen and thawed, it is necessary to pass the lipid phase transition temperature rapidly.

In conclusion, the present study indicates the possibility that the different color tone in the immature oocytes from prepubertal and adult minke whales was caused by the difference in the spatial pattern of lipid droplets. It was also shown that the lipid contents in vitrified-warmed minke whale oocytes were similarly high when compared with vitrified-warmed porcine oocytes. Therefore, it seems that minke whale oocytes, as well as porcine oocytes, are sensitive to freezing or vitrification.

\section{References}

1. Fukui $\mathbf{Y}$, Mogoe $\mathbf{T}$, Ishikawa $\mathbf{H}$, Ohsumi S. Factors affecting in vitro maturation of minke whale (balaenoptera acutorostrata) follicular oocytes. Biol Reprod 1997; 56: 523-528.

2. Fukui $Y$, Mogoe $T$, Ishikawa $H$, Ohsumi S. In vitro fertilization of in vitro matured minke whale (balaenoptera acutorostrata) follicular oocytes. Mar Mamm Sci 1997; 13: 395-404.

3. Asada M, Horii M, Mogoe T, Fukui Y, Ishikawa $H$, Ohsumi S. In vitro maturation and ultrastructural observation of cryopreserved minke whale (balaenoptera acutorostrata) follicular oocytes. Biol Reprod 2000; 62: 253-259.

4. Asada M, Tetsuka M, Ishikawa H, Ohsumi S, Fukui Y. Improvement on in vitro maturation, fertilization and development of minke whale (balaenoptera acutorostrata) oocytes. Theriogenology 2001; 56: 521-533.

5. Homa ST, Racowsky C, McGaughey RW. Lipid analysis of immature pig oocytes. J Reprod Fertil 1986; 77: 425-434.

6. McEvoy TG, Coull GD, Speake BK, Staines ME, Broadbent PJ. Estimation of lipid and fatty acid composition of zona-intact pig oocytes. I Reprod Fertil Abstract Series 1997; 20: 10.

7. McEvoy TG, Coull GD, Broadbent PJ, Hutchinson JS, Speake BK. Fatty acid composition of lipids in immature cattle, pig and sheep oocytes with intact zona pellucida. J Reprod Fertil 2000; 118: 163-170.

8. Kim JY, Kinoshita M, Ohnishi M, Fukui Y. Lipid and fatty acid analysis of fresh and frozen-thawed immature and in vitro matured bovine oocytes. Reproduction 2001; 122: 131-138.

9. Didion BA, Pomp D, Martin MJ, Homanics GE, Markert CL. Observations on the cooling and cryopreservation of pig oocytes at the germinal vesicle stage. J Anim Sci 1990; 68: 2803-2810.

10. Liebermann J, Nawroth F, Isachenko V, Isachenko E, Rahimi G, Tucker MJ. Potential importance of vitrification in reproductive medicine. Biol Reprod 2002; 67: 1671-1680.

11. O'Brien JK, Dwarte D, Ryan JP, Maxwell WMC, Evans G. Developmental capacity, energy metabolism and ultrastructure of mature oocytes from prepubertal and adult sheep. Reprod Fertil Dev 1996; 8: 1029-1037.

12. Marchal R, Feugang JM, Perreau C, Venturi E, Terqui $\mathbf{M}$, Mermillod $\mathbf{P}$. Meiotic and developmental competence of prepubertal and adult swine oocytes. Theriogenology 2001; 56: 17-29.

13. Khatir $\mathbf{H}$, Lonergan $\mathbf{P}$, Carolan $\mathbf{C}$, Mermillod $\mathbf{P}$. Prepubertal bovine oocyte: a negative model for studying oocyte developmental competence. Mol Reprod Dev 1996; 45: 231-239.

14. Revel F, Mermillod P, Peynot N, Renard JP, Heyman Y. Low developmental capacity of in vitro matured and fertilized oocytes from calves compared with that of cows. J Reprod Fertil 1995; 103: 115-120.

15. Bligh EG, Dyer WJ. A rapid method of total lipid extraction and purification. Can J Biochem Physiol 1959; 39: 911-917. 
16. Sathananthan AH, Kirby C, Trounson A, Philipatos D, Shaw J. The effects of cooling mouse oocytes. J Assist Reprod Genet 1992; 9: 139-148.

17. Sathananthan AH. Ultrastructural changes during meiotic maturation in mammalian oocytes: unique aspects of the human oocyte. Microsc Res Tech 1994; 27: $145-164$.

18. Casella JF, Flanagan MD, Lin S. Cytochalasin D inhibits actin polymerization and induces depolymerization of actin filaments formed during platelet shape change. Nature 1981; 293: 302-305.

19. Nagashima H, Kashiwazaki N, Ashman RJ, Grupen CG, Seamark RF, Nottle MB. Removal of cytoplasmic lipid enhances the tolerance of porcine embryos to chilling. Biol Reprod 1994; 51: 618-622.

20. Nagashima H, Kashiwazaki N, Ashman RJ, Grupen CG, Nottle MB. Cryopreservation of porcine embryos. Nature 1995; 374: 416.

21. Alberts B, Bray D, Lewis J, Raff M, Roberts K, Watson JD. Molecular Biology of the Cell. Third edition 1995; 659: Newton Press.

22. Ferguson EM, Leese HJ. Triglyceride content of bovine oocytes and early embryos. J Reprod Fertil 1999; 116: 373-378.

23. Hyun SH, Lee GS, Kim DY, Kim HS, Lee SH, Kim S, Lee ES, Lim JM, Kang SK, Lee BC, Hwang WS. Effect of maturation media and oocytes derived from sows or gilts on the development of cloned pig embryos. Theriogenology 2003; 59: 1641-1649.

24. Isachenko $\mathrm{V}$, Isachenko $\mathrm{E}$, Michelmann HW, Alabart JL, Vazquez I, Bezugly N, Nawroth F. Lipolysis and ultrastructural changes of intracellular lipid vesicles after cooling of bovine and porcine GV-oocytes. Anat Histol Embryol 2001; 30: 333-338.

25. Arav A, Zeron Y, Leslie SB, Behboodi E, Anderson GB, Crowe JH. Phase transition temperature and chilling sensitivity of bovine oocytes. Cryobiology 1996; 33: 589-599. 